Less is More I: a pessimistic view of piecewise smooth bifurcation theory

Glendinning, Paul

2016

MIMS EPrint: 2016.20

Manchester Institute for Mathematical Sciences

School of Mathematics

The University of Manchester

\footnotetext{
Reports available from: http://eprints.maths.manchester.ac.uk/

And by contacting: The MIMS Secretary

School of Mathematics

The University of Manchester

Manchester, M13 9PL, UK
} 


\title{
Less is More I: a pessimistic view of piecewise smooth bifurcation theory
}

\author{
Paul Glendinning \\ School of Mathematics, University of Manchester, Oxford Road, Manchester M13 9PL, U.K. \\ p.a.glendinning@manchester.ac.uk \\ I am grateful to Mike Jeffrey and Rachel Kuske for conversations that helped crystallize these ideas, and to the \\ Simon Foundation for support at the CRM, Barcelona.
}

\begin{abstract}
The analysis of piecewise smooth bifurcations reveals an alarming proliferation of cases as the dimension of phase space increases. This suggests that a different approach needs to be taken when trying to describe bifurcations. In particular, it may not be helpful to analyze particular bifurcations at the level of detail that is standard for smooth systems.
\end{abstract}

\section{Introduction}

"Can you do addition?" the White Queen asked. "What's one and one and one and one and one and one and one and one and one and one?" "I don't know," said Alice. "I lost count." [2] chapter 9.

With the analysis of more bifurcations of piecewise smooth (PWS) systems it is becoming clear that there is a proliferation of cases as the dimension of the ambient phase space increases. In smooth dynamical systems the centre manifold theorem implies that the range of typical local bifurcations is severely restricted and independent of the phase space dimension. Indeed, only the saddle-node bifurcation and Hopf bifurcation are generic, though the addition of symmetry or other special features can add complications. The global bifurcations of typical smooth systems are also constrained, although features such as Shilnikov's Theorems for homoclinic orbits, and the possibility of bifurcations being dense in parameter space mean that it may be impossible to give a complete description. Nonetheless, there are robust features common to all these bifurcations that can be described sensibly.

The situation for PWS systems appears significantly harder to deal with. Whereas typical smooth systems have a manageable number of fundamental bifurcations, the number of cases for PWS systems increases with the dimension of the phase space in such a way that a complete classification would require the enumeration of an infinite set of possibilities. This may be good for the production of academic papers, but it does not necessarily help us to understand potential applications.

These observations suggest that the attitude to bifurcations of PWS systems needs to be somewhat different from that applied to smooth systems. In particular, it may be more useful to develop weaker results which apply quite generally rather than give a complete picture of the bifurcations that can occur (a list that might take forever). Such results might provide a rather less detailed description of possibilities, or the development of techniques that would allow a more detailed description if the occasion (i.e. the application) arose, but would not attempt to apply these methods to all possible

Research Perspectives CRM Barcelona, Spring 2016, vol. 9, in Trends in Mathematics Springer-Birkhuser, Basel 
situations without further motivation. This is the 'Less is More' philosophy of the title, described in greater detail in $[\mathbf{1 0}]$.

In the remainder of this note we give examples of the proliferation described above.

\section{Transitions to chaos in PWS maps}

For smooth maps of the interval, for example the quadratic map, the transition to chaos is very easy to characterize. Let $\mathcal{P}$ be the set of periods for the map, i.e. if the map is $f$ then $p \in \mathcal{P}$ if and only if $f$ has a periodic orbit of least period $p$. It follows from the proof of Sharkovskii's Theorem that for any continuous non-chaotic map of the interval

$$
\mathcal{P}=\left\{2^{n}, \quad 0 \leq n \leq N\right\}
$$

for some $N \in\{0,1,2, \ldots\} \cup\{\infty\}$. To some extent this explains why period-doubling cascades are so ubiquitous, though it also holds for Nordmark's continuous square root map where the stable periodic orbits form a period-adding sequence.

For PWS maps with discontinuities the situation is more complicated. If we consider maps with a single discontinuity and with two increasing continuous branches then Gambaudo et al [5] show that the only infinite sets of periods that can occur on the boundary of chaos are arbitrary period multiplying

$$
\mathcal{P}=\left\{p_{n} \mid p_{n+1}=a_{n} p_{n} \quad a_{n} \in \mathbb{N}, a_{n} \geq 2\right\}
$$

though these correspond to points on a one-parameter boundary of chaos in a twoparameter space; typically the transition to chaos occurs after a finite number of periods are created.

In the more general case of PWS maps with a single discontinuity and two continuous monotonic branches at least one of which is decreasing there is a new robust route to chaos involving creation of infinitely many periodic orbits: the anharmonic route $[6]$. In the case of one increasing and one decreasing branch this generates periods

$$
\mathcal{P}=\left\{p_{n} \mid p_{n+1}=2 p_{n}+(-1)^{n}\right\}
$$

and more general forms are also possible involving higher iterates of the map or maps with two decreasing branches.

Furthermore, if we consider all possible sets of periodic orbits for non-chaotic maps then the set of infinite possible periods on the boundary of chaos, ignoring the multiplying of (1), is given by a subshift of finite type corresponding to different sequences of renormalizations or induced maps [7].

These results show that even though the continuous case is constrained, the set of things that can happen in the discontinuous case is complicated by a proliferation from essentially one to uncountably many possibilities. Moreover, whilst these can be characterized it is not clear which are relevant for the examples that arise in PWS dynamics (which possibilities occur in expanding maps, or in piecewise linear maps?). The theory that makes this analysis possible was developed in the late 1970s [12] and these ideas are worth revisiting.

\section{The border collision normal form}

Now consider continuous PWS maps in dimension greater than one. Suppose that phase space is divided into two regions by a switching surface and smooth maps are defined on 
each side of the surface, and the PWS map is continuous but not differentiable across the surface. Suppose a fixed point exists at some parameter value in one region, and as parameters vary it moves to intersect the switching surface. What happens?

Nusse and Yorke [13] show that given some genericity conditions the bifurcation is described locally by the border collision normal form, with leading order terms

$$
z_{n+1}=F\left(z_{n}\right)= \begin{cases}A_{0} z_{n}+m & \text { if } x_{1}<0 \\ A_{1} z_{n}+m & \text { if } x_{1}>0\end{cases}
$$

with $A_{0}=\left(\begin{array}{cc}t_{0} & 1 \\ -d_{0} & 0\end{array}\right), A_{1}=\left(\begin{array}{cc}t_{1} & 1 \\ -d_{1} & 0\end{array}\right)$ and $m=\mu(1,0)^{T}$.

This can be generalized to $\mathbb{R}^{n}$ where the map takes the same form and provided some simple genericity conditions hold the matrices $A_{i}$ can be written in observer canonical form where the first column is arbitrary and the remainder has ones on the upper offdiagonal and zeroes everywhere else, and $m=\mu(1,0, \ldots, 0)^{T}[\mathbf{3}]$.

Using some beautiful technical results of Buzzi and Tsujii $[\mathbf{1}, \mathbf{1 4}]$ it is possible to prove bifurcations from fixed points to $n$-dimensional attractors in these maps.

Theorem 1 ([8, 9]) Consider the border collision normal form in $\mathbb{R}^{n}(n=2, \ldots)$. There exist open regions of parameter space $B_{n}$ such that for each parameter in $B_{n}$ if $\mu<0$ then the border collision normal form has a stable fixed point; whilst if $\mu>0$ then the border collision normal form has at least one attractor with an invariant measure absolutely continuous with respect to $n$-dimensional Lebesgue measure. If $n=2$ then the attractor has topological dimension equal to 2 . If $n>2$ then the attractor has Hausdorff dimension equal to $n$ and generically has topological dimension equal to $n$.

What about other transitions: can we go from attractors of any given Hausdorff dimension to any other Hausdorff dimension? If so, is this the right way of looking at the problem? If not, how much is the dynamics constrained and how should this be described?

\section{Boundary Equilibrium Bifurcations}

Boundary equilibrium bifurcations (BEBs) occur if a stationary point of a PWS system intersects the boundary of one of the regions on which the smooth components of the system are defined. Even in planar flows Filippov states that there are 12 cases that need to be considered [4]. The example below of a flow in $\mathbb{R}^{3}$ shows just how complicated the bifurcation can become in higher dimensions. This example has a Shilnikov homoclinic orbit with a sliding section immediately after the bifurcation, and hence all the levels of complexity of this three-dimensional flow $[\mathbf{1 1}]$.

The example uses two differential equations with switching surface $z=0$, so $F^{+}$ defines the flow if $z>0$ and $F^{-}$defines the flow if $z<0$, where

$$
F^{+}(\mathbf{x}, \nu)=\left(\begin{array}{ccc}
-\rho & a & -\omega \\
0 & \lambda & 0 \\
\omega & b & -\rho
\end{array}\right)\left(\begin{array}{c}
x \\
y \\
z-\nu
\end{array}\right), \quad F^{-}(\mathbf{x}, \nu)=\left(U_{1}, U_{2}, U_{3}\right)^{T},
$$

with $U_{3}>0$ and $U_{k} \neq 0, k=1,2$. The upper flow $F^{+}$has a stationary point at $(0,0, \nu)$ so there is a BEB if $\nu=0$. Suppose that the remaining constants have been fixed, except $\omega$ which will be used to explore the sensitivity of the bifurcation to changes in the other 
parameters. Consider the case $\nu>0$. By scaling we may set $\nu=1$ and then the final parameter $\omega$ can be used to determine the different dynamics that can occur.

The stationary point $(0,0,1)$ has eigenvalues $\lambda$ and $-\rho \pm i \omega$ which may be chosen so that $\frac{\rho}{\lambda}<1$. Thus if there is a homoclinic orbit, i.e. an orbit which approaches $(0,0,1)$ in both forwards and backwards time, the classic results of Shilnikov proving the existence of chaos hold with minor technological modifications to take the sliding section into account $[\mathbf{1 1}]$. Numerical simulations $[\mathbf{1 1}]$ show that $\omega$ can be chosen so that the flow includes just such a homoclinic orbit and hence that the dynamics in $\nu>0$ of this $\mathrm{BEB}$ is determined by a family of differential equations (as the other parameters vary) which has complicated bifurcation structure itself.

\section{Conclusion}

A subject comes of age when the extent and scope of the discipline is generally accepted. It is time to grow up. What features should define a useful bifurcation theory in piecewise smooth dynamics? An attempt to answer this question is made in [10] - here we have simply shown that a complete classification can lead to very long lists. Of course there are many other issues (e.g. noise and nonlinearity) not mentioned here, but the fundamental problem is: how do we select methods and results to avoid endless lists?

\section{References}

[1] J. Buzzi (1999) Absolutely continuous invariant measures for generic multi-dimensional piecewise affine expanding maps, Int. J. Bifn. E Chaos 9 1743-1750.

[2] L. Carroll (1871) Through the Looking-Glass, and What Alice Found There, Macmillan.

[3] M. di Bernardo (2003) Normal forms of border collision in high dimensional non-smooth maps, Proc. IEEE ISCAS $2003 \mathbf{3} 76-79$.

[4] A.F. Filippov (1988) Differential Equations with discontinuous right hand sides, Kluwer, Netherlands.

[5] J.M. Gambaudo, I. Procaccia, S. Thomae and C. Tresser (1986) New universal scenarios for the onset of chaos in Lorenz type flows, Phys. Rev. Lett. 57 925-928.

[6] P. Glendinning (1993) The anharmonic route to chaos: kneading theory, Nonlinearity 6 349-367.

[7] P. Glendinning (2014) Renormalization for the boundary of chaos in piecewise monotonic maps with a single discontinuity, Nonlinearity 27 R143-R162.

[8] P. Glendinning (2015) Bifurcation from stable fixed point to $N$-dimensional attractor in the border collision normal form, Nonlinearity 28 3457-3464.

[9] Glendinning P (2016) Bifurcation from stable fixed point to two-dimensional attractor in the border collision normal form, IMA J. Appl. Math. doi:10.1093/imamat/hxw001 (12 pages).

[10] P. Glendinning (2016) Less is More II: an optimistic view of piecewise smooth bifurcation theory, this volume.

[11] P. Glendinning (2016) Shilnikov chaos, Filippov sliding and Boundary Equilibrium Bifurcations, in preparation.

[12] J. Milnor and W. Thurston (1988) On iterated maps of the interval, in Dynamical systems, LNM 1342, 465-563, Springer, Berlin.

[13] H.E. Nusse and J.A. Yorke (1992) Border collision bifurcations including period two to period three bifurcation for piecewise smooth systems, Physica D 57 39-57.

[14] M. Tsujii (2001) Absolutely continuous invariant measures for expanding piecewise linear maps, Invent. Math. 143 349-373. 